Revue des patrimoines

$31 \mid 2017$

Patrimoines de la santé : essais de définition - enjeux de conservation

\title{
Protéger le patrimoine hospitalier au titre des monuments historiques
}

Bilan et perspectives

Protecting the hospital heritage as historic monuments: results and perspectives

Dominique Perrin et Frantz Schœnstein

\section{(2) OpenEdition \\ Journals}

Édition électronique

URL : http://journals.openedition.org/insitu/14149

DOI : 10.4000/insitu. 14149

ISSN : $1630-7305$

Éditeur

Ministère de la Culture

Référence électronique

Dominique Perrin et Frantz Schœenstein, « Protéger le patrimoine hospitalier au titre des monuments

historiques », In Situ [En ligne], 31 | 2017, mis en ligne le 28 février 2017, consulté le 09 octobre 2020.

URL : http://journals.openedition.org/insitu/14149 ; DOI : https://doi.org/10.4000/insitu.14149

Ce document a été généré automatiquement le 9 octobre 2020.

\section{c) $(\$)$}

In Situ Revues des patrimoines est mis à disposition selon les termes de la licence Creative Commons Attribution - Pas d'Utilisation Commerciale - Pas de Modification 4.0 International. 


\section{Protéger le patrimoine hospitalier au titre des monuments historiques}

Bilan et perspectives

Protecting the hospital heritage as historic monuments: results and perspectives

Dominique Perrin et Frantz Schœnstein

1 Même s'il n'est alors pas reconnu en tant que tel, le patrimoine hospitalier est présent dans la première liste des monuments historiques publiée en France, en 1840, avec l'inscription de «l'hôtel-Dieu» d'Angers. Comme pour la plupart des catégories de patrimoine, le champ de la protection s'est élargi au fil du temps, tant en termes thématiques que chronologiques.

2 Mais dans un contexte de redéfinition du périmètre de l'action publique et de révision des cartes des services publics, la question de la préservation des infrastructures hospitalières patrimoniales se pose avec une acuité et une ampleur nouvelles.

3 De nouveaux critères et de nouveaux principes de protection, susceptibles de mieux accompagner la nécessaire adaptation de l'usage de certains édifices, sont donc à mettre en œuvre - et, dans une large mesure, à inventer.

\section{Le classement des hôpitaux et hospices : évolution dans le temps des motifs de la protection}

4 Il s'agit, sous ce titre, de procéder à un bilan des mesures de classement au titre des monuments historiques existantes, concernant les hôpitaux et les hospices, en vue notamment de dégager les motifs d'une telle protection.

Le thème du présent numéro d'In Situ porte sur la période qui va de l'époque moderne à nos jours. Toutefois, il n'est guère pensable de procéder à un traitement cohérent de notre sujet en ne tenant compte que des édifices d'une époque donnée. Aussi la présente étude concerne-t-elle l'ensemble des bâtiments hospitaliers construits en France depuis le Moyen Âge jusqu'à l'époque contemporaine. 
6 La législation applicable aux monuments historiques est comprise dans le Code du patrimoine et découle des dispositions de la loi du 31 décembre 1913 sur les monuments historiques. Il est précisé que sont classés au titre des monuments historiques les immeubles dont la conservation présente, au point de vue de l'histoire ou de l'art, un intérêt public. Les immeubles qui, sans justifier une demande de classement immédiat, présentent un intérêt historique ou artistique suffisants pour en rendre désirable la préservation peuvent être inscrits au titre des monuments historiques.

7 Il s'agit donc de se demander quels sont les établissements hospitaliers dont la conservation a été considérée comme présentant un intérêt public, et quels ont été les critères retenus. Sur cent ans, ces critères ont bien sûr évolué.

8 Nous incluons dans notre étude les institutions destinées à héberger les mendiants, qui se développèrent au XVII e siècle. À la suite de la déclaration du 14 juin 1662, obligation fut faite d'établir dans chaque grande ville du royaume un établissement de ce type, sous le nom d'hôpital général. Il s'agissait avant tout de lieux d'enfermement et non d'établissements de soins. Destinés au départ à l'accueil des indigents valides, les lieux accueillirent en fait, outre les orphelins et les vieillards sans revenus, de vrais malades, comme les aliénés.

\section{La liste du Journal officiel du 18 avril 1914}

9 La loi du 31 décembre 1913 sur les monuments historiques prévoit l'établissement et la publication au Journal officiel d'une liste des immeubles considérés comme classés avant sa promulgation. Cette liste a été publiée au JO du 18 avril 1914. Les protections les plus anciennes qui y figurent remontent à la liste de 1840, résultant des propositions à la commission des Monuments historiques établies par les préfets pour l'affectation des crédits de restauration.

10 La liste de 1914 mentionne d'abord un élément remarquable d'un établissement pour pèlerins fondé dans la seconde moitié du XII ${ }^{e}$ siècle, d'esprit encore largement roman, le " passage de l'hôpital » à Pons (Charente-Maritime), qui avait été classé par arrêté du 8 octobre 1879. Elle mentionne également l'hôpital Saint-Jean à Angers (Maine-etLoire), figurant dans la liste de 1840 sous l'appellation d'hôtel-Dieu, dont la grande salle des malades gothique, du XII ${ }^{\mathrm{e}}$ siècle, abrite maintenant la tenture Le chant $d u$ monde de Jean Lurçat.

11 Figurent également sur la liste de 1914 l'ancienne salle des malades de l'hôpital de Tonnerre (Yonne), de la fin du XIII ${ }^{e}$ siècle, ainsi que l'hôtel-Dieu de Beaune (Côte-d'Or), $\mathrm{du} \mathrm{xv}^{\mathrm{e}}$ siècle, l'origine de la protection remontant dans les deux cas à la liste de 1862. Pour l'hôpital d'Issoudun (Indre), nous avons une protection progressive avant 1914: sur la liste de 1862, figure la mention suivante: «Arbre de Jessé, dans la chapelle de l'hôpital à Issoudun ». Il s'agit en fait de deux représentations de l'arbre de Jessé, sculptées sur les parois de la chapelle de l'établissement. Par arrêté du 19 mars 1908, est classée la chapelle Saint-Roch de l'ancien hôtel-Dieu, dont les arbres de Jessé font partie. La salle des malades l'est le 8 octobre 1910.

12 Le 9 juin 1904, les restes de l'ancien hôpital des Baux-de-Provence (simples «murs et maisons des Baux » dans la liste de 1862) sont spécifiquement classés en application de la loi du 30 mars 1887; ce classement est repris dans la liste de 1914 (il s'agit 
probablement de l'hôpital Quiqueran, du $\mathrm{XVI}^{\mathrm{e}}$ siècle). Y figurent enfin deux autres ensembles postérieurs au Moyen Âge, à savoir l'hôtel des Invalides à Paris et l'hôpital de Carpentras (Vaucluse), du XVIII siècle, tous deux mentionnés dans la liste de 1862.

Quelles conclusions pouvons-nous tirer de ces mentions dans la liste de 1914 ? Les constructions médiévales occupent une large part, les parties les plus remarquables étant dans certains cas privilégiées, en négligeant l'organisation d'ensemble, comme c'est le cas à Pons. Ainsi, seul le passage voûté de l'établissement pour pèlerins a été classé dans un premier temps. Tout en enjambant la route menant à Saint-Jacques-deCompostelle, il reliait en fait la chapelle actuellement ruinée à la salle des pèlerins encore conservée, bien que transformée. Après des mesures d'inscription intermédiaires, ce sont tous les principaux éléments ou vestiges du dispositif médiéval qui seront finalement classés le 16 juin 1998 (salle des pèlerins, vestiges de l'église, sols). Deux logis sont inscrits, dont un datant du XVIII siècle, protégé pour ses façades et ses toitures ${ }^{1}$.

Même évolution pour l'hôpital d'Issoudun : tous les bâtiments anciens, allant du $\mathrm{xv}^{\mathrm{e}}$ siècle au XVIII ${ }^{e}$ siècle, sont classés par arrêté du 9 septembre 1965, y compris, est-il précisé, la chapelle, la salle des malades et le sol de la cour. Un musée est actuellement aménagé dans les lieux ${ }^{2}$. Citons un cas extrême: de l'ensemble de l'hôtel-Dieu de Troyes, fruit d'une reconstruction au XVIII ${ }^{\mathrm{e}}$ siècle, seule la grille d'entrée, splendide, il est vrai, est mentionnée dans la liste de 1914. Il faut attendre le 23 novembre 1964 pour voir classée l'intégralité des façades et toitures des bâtiments de la cour d'honneur, avec les ailes en retour sur la rue de la Cité, de la chapelle et de la pharmacie ${ }^{3}$.

15 «L'équivalent » de la salle des malades de l'hôpital Saint-Jean d'Angers, à savoir l'église Sainte-Jeanne-d'Arc du Mans (Sarthe), qui est en réalité l'ancienne salle des malades de Coëffort, n'est classée qu'en 1947. Les deux salles présentent de grandes similitudes en dimensions et en organisation (trois vaisseaux couverts de croisées d'ogives sont déterminés par deux files de colonnes). Ces deux exemples témoignent d'un programme hospitalier voulu par les Plantagenêts, la situation à la périphérie des villes n'étant peut-être pas sans rapport avec des préoccupations liées à l'hygiène.

Venons-en maintenant aux seuls bâtiments cités dans la liste de 1914 pour la période des $\mathrm{XVII}^{\mathrm{e}}$ et $\mathrm{XVIII}^{\mathrm{e}}$ siècles. Le classement de l'hôtel national des Invalides découle incontestablement de son grand intérêt historique et architectural. Mais l'édifice marque aussi la naissance d'un établissement spécialisé dans l'accueil des vétérans âgés ou infirmes. Les soins s'adressaient aux seuls pensionnaires. La mention de l'hôtel-Dieu de Carpentras est particulièrement intéressante, car il s'agit là d'un édifice du XVIII ${ }^{\mathrm{e}}$ siècle, cas très rare dans les protections $d u$ XIX ${ }^{e}$ siècle. Le plan est attribué à l'architecte Antoine d'Allemand, de Carpentras, qui n'a pas une renommée internationale. Mais cet édifice, situé en terre pontificale, brille par la qualité de son exécution et de sa conception, due notamment à la personnalité de son commanditaire, Monseigneur d'Inguimbert ${ }^{4}$. [Lien vers article sur l'hôtel-Dieu de Carpentras, In situ, Patrimoines de la santé]

\section{L'évolution de l'application de la loi de 1913}

17 Entre les deux guerres mondiales, d'autres édifices médiévaux sont pris en compte par un classement, comme par exemple la salle basse de l'ancien hôtel-Dieu de Laon 
(Aisne), dite salle des Chanoines, à la belle voûte gothique, classée le 6 mars 1928. Le classement complet de l'hôtel-Dieu intervient en 1942.

En ce qui concerne les protections en totalité, est classé le 8 juin 1928 l'ancien hôtelDieu de Dole (Jura), entrepris au début du XvII e siècle, qui s'ordonne autour d'une cour carrée. Le classement de l'ancien hôpital de Hautefort (Dordogne), le 27 août 1931, s'applique à un ensemble du XVII ${ }^{e}$ siècle dont le plan est en croix grecque ${ }^{5}$. Le 15 juin 1932 est classé l'hôpital Notre-Dame à Seclin (Nord), fondé en 1246 par Marguerite de Flandre, et dont l'état actuel découle d'une succession de campagnes de travaux ultérieures.

19 À partir de la Seconde Guerre mondiale, les mesures de classement s'intensifient. Pour la seule décennie 1940-1950, on peut citer quatre mesures de classement en totalité, pour l'ancien hôpital de la Charité à Senlis (Oise, XVIII siècle) en janvier 1942, l'hôpital de la Charité à Langres (Haute-Marne, XVIII ${ }^{\mathrm{e}}$ siècle) en décembre 1944, l'hôpital général de Valenciennes (Nord, XVIII siècle) en juin 1945 et l'ancien hôtel-Dieu de Bourges $(\text { Cher })^{6}$ pour les bâtiments des XVI ${ }^{e}$ et XVIII ${ }^{e}$ siècles, en juin 1946.

20 Notons le passage de l'inscription (qui datait de 1927) au classement en totalité, le 29 janvier 1951, de la Vieille Charité de Marseille, faisant fonction d'hôpital général. Ce chef-d'œuvre de Pierre Puget, remarqué par Le Corbusier, comporte une chapelle disposée de façon exceptionnelle, totalement isolée au centre de la cour, rappelant que les hôpitaux généraux étaient au départ destinés à des indigents, le plus souvent valides, ayant des activités, et que l'on est en dehors du schéma de salles de malades ouvertes vers la chapelle.

21 Plusieurs autres ensembles font l'objet d'un classement après la Seconde Guerre mondiale, mais nous nous arrêterons là dans une énumération chronologique qui pourrait devenir fastidieuse, et il est temps de tirer des conclusions de l'ensemble du corpus existant de nos jours.

Relevons préalablement que plusieurs établissements bénéficient de protections mixtes, étant en partie classés, en partie inscrits. La liste de 1914 ne mentionnait que la chapelle dite Sainte-Croix-de-Jérusalem, dans l'hôpital général de Dijon. De nombreux autres éléments ont été inscrits en $2007^{7}$. Seules la chapelle et l'apothicairerie de l'hôpital de Cluny (Saône-et-Loire) sont classées, le 3 mai 2002, tout le reste étant inscrit depuis $2001^{8}$. Parfois, la combinaison des deux niveaux de protection peut s'avérer complexe, comme c'est le cas pour l'hospice de La Grave à Toulouse (HauteGaronne) $)^{9}$.

De façon générale, les classements existants concernent surtout des ensembles antérieurs à la Révolution.

Certains de ces ensembles étaient spécialisés dans un type d'affection contagieuse, devenant de fait des lieux d'exclusion. Il reste peu de témoignages des très nombreuses léproseries qui couvraient le territoire, se résumant souvent aux seules chapelles de ces établissements, comme celle du Petit-Quevilly (Seine-Maritime) figurant sur la liste de 1862. C'est un ensemble plus important qui subsiste au lieu-dit Voisinlieu à Beauvais (Oise), classé en 1939 pour ses éléments les plus significatifs, une inscription complémentaire étant intervenue en $1989^{10}$. L'hôpital Saint-Louis de Paris, fondé par Henri IV, est classé en grande partie, d'autres éléments étant inscrits. Destiné aux pestiférés, c'était en fait un lazaret ${ }^{11}$. 
En revanche, on peut constater que peu d'édifices du xix siècle, et encore moins $d u x^{e}$ siècle, ont fait l'objet de mesures de classement.

Lorsque l'on entreprend, dans la base Mérimée, une recherche sur les édifices classés datant dans leur ensemble du XIX ${ }^{e}$ siècle, en entrant le mot «hôpital », nous obtenons pour la métropole trois résultats : l'ancien hospice Saint-Charles à Rosny-sur-Seine (Yvelines), et deux établissements pour aliénés, ceux du Mans (Sarthe) et de SaintMaurice (Val-de-Marne). Nous écartons l'hôpital Sainte-Marie à Angers, qui bénéficie d'un classement depuis le 23 septembre 1930, mais uniquement pour les peintures murales de sa chapelle.

L'ancien hospice Saint-Charles de Rosny, œuvre de Joseph-Antoine Frœlicher, a été fondé par la duchesse de Berry, et comprend une cour organisée autour d'une chapelle contenant le cœur de son mari assassiné en 1820 . On peut supposer que le classement répond moins à l'intérêt de la typologie hospitalière qu'à l'aspect historique de la chapelle, de type mémoriel, et à la qualité de son décor néoclassique.

Les deux autres exemples sont des hôpitaux psychiatriques.

Les établissements pour les aliénés se développent au XIX ${ }^{\mathrm{e}}$ siècle. L'asile d'aliénés du Mans est classé en totalité pour sa chapelle et son pavillon des agités, les autres bâtiments l'étant pour leurs seules façades et toitures, par arrêté du 4 octobre 2001. L'hôpital Esquirol à Saint-Maurice (Val-de-Marne) est classé en totalité pour sa seule chapelle, tous les autres bâtiments étant classés pour leurs façades et toitures, avec leurs galeries et portiques, leurs escaliers et leurs terrasses.

L'asile du Mans, construit par Pierre-Félix Delarue et dont la création remonte à 1828, reprend le plan en peigne dit de l'Académie ${ }^{12}$. Attardons-nous quelques instants sur ce type de plan. On sait que l'incendie de l'Hôtel-Dieu de Paris en 1772 suscita nombre de projets pour sa reconstruction. Finalement, l'Académie des sciences, sollicitée, fit établir le plan de l'hôpital idéal inspiré de celui de Plymouth, en Angleterre: des pavillons allongés, disposés de part et d'autre d'une cour, sont reliés de façon transversale par une galerie, déterminant un schéma en peigne. Ce plan aura après la Révolution un grand succès ${ }^{13}$. (voir article de M. Lauro, même numéro)

La reconstruction de l'asile de Charenton débute en 1838. L'architecte Émile-Jacques Gilbert appliqua de façon plus systématique les idées du médecin Jean-Étienne Esquirol, lui-même élève de Philippe Pinel. De part et d'autre de l'axe défini par le bâtiment de l'administration et la chapelle se répartissent les quartiers réservés d'une part aux hommes, d'autre part aux femmes. Le module de base est formé par des bâtiments disposés en $U$, le quatrième côté, au sud, étant fermé par une galerie. En outre, ce magnifique ensemble est marqué par les références à l'antique. La composition générale en terrasse est inspirée de celle du temple de la Fortune à Palestrina (Latium), en Italie. L'hôpital fut achevé par Arthur Diet en $1886^{14}$.

Notons que le 17 décembre 1996, la commission régionale du patrimoine historique, archéologique et ethnologique (COREPHAE) de Bretagne a examiné le dossier de l'hôpital Frémeur, du xvI ${ }^{e}$ siècle, à Quimperlé (Finistère) et a émis un avis favorable au classement de cet ensemble avec sa chapelle, classement qui s'est concrétisé (arrêté du 24 mai 2004) après l'examen du dossier par la commission supérieure des Monuments historiques. Par contre, la commission régionale a rejeté l'inscription de l'hôpital SaintMichel, construit à côté autour de 1900. De type pavillonnaire, il comprenait des voûtes 
en berceau brisé, conformément au système mis au point par l'ingénieur Casimir Tollet. Ces voûtes étaient munies à leur faîte d'orifices censés évacuer l'air vicié ${ }^{15}$.

Pour le $\mathrm{XIX}^{\mathrm{e}}$ siècle, mais hors métropole, l'ensemble de l'ancienne léproserie de l'Acarouany, fondée en 1836 par la révérende mère Javouhey à Mana, en Guyane, constitue un autre exemple d'établissement spécialisé classé (arrêté du 20 décembre 1999) ${ }^{16}$. La Guyane est d'ailleurs bien dotée d'hôpitaux classés de l'époque moderne, car outre cet exemple, figurent l'hôpital André-Bouron à Saint-Laurent-du-Maroni (arrêté du 9 mars 1999) ${ }^{17}$ et l'hôpital Jean-Martial à Cayenne (arrêté du 29 octobre 2012) ${ }^{18}$.

Pour le $\mathrm{xx}^{\mathrm{e}}$ siècle, la réponse est simple : nous avons un seul exemple d'hôpital construit entièrement à cette époque et classé au titre des monuments historiques, l'hôpitalmémorial France-États-Unis de Saint-Lô. Sont ainsi classés par arrêté du 24 septembre 2008 les façades et les toitures de l'hôpital d'origine, le hall d'entrée, les deux couloirs de circulation avec les patios intérieurs, la salle d'opération du premier étage, la mosaïque de Fernand Léger et les façades et toitures du pavillon d'entrée. Ce nouvel hôpital a été construit après la dévastation de la ville de Saint-Lô en 1944. Il est doublé d'un hospice, financé pour moitié par des fonds collectés aux États-Unis par l'American Aid to France. L'hôpital-bloc à circulation verticale, réalisé sur les plans de l'Américain Paul Nelson, est inauguré en 1954. Les espaces techniques sont réunis sur un plateau horizontal bas formant socle à la partie dédiée à l'hospitalisation proprement dite. Cet établissement eut un grand impact sur l'architecture hospitalière de la seconde moitié $\mathrm{du} \mathrm{xx}^{\mathrm{e}}$ siècle (voir article sur Arles d'Éléonore Marantz). De plus, une mosaïque de Fernand Léger, célébrant les liens entre la France et les États-Unis, rappelle la fonction également mémorielle du bâtiment ${ }^{19}$.

Parmi les parties classées de l'hôpital de Saint-Lô qui viennent d'être énumérées figure une salle d'opération. C'est l'occasion de signaler que l'hôpital Saint-Jacques de Besançon conserve, de nos jours, un bloc opératoire dénommé Saint-Joseph, comprenant deux salles d'opération, témoignage exceptionnellement conservé du système conçu par l'ingénieur André Walter, mis en service dans cet établissement en $1959^{20}$. La lumière d'un grand projecteur placé à l'extérieur était diffusée par réflexion sur une demie-voûte elliptique surmontant la table d'opération, d'où l'absence dans la salle de chaleur liée à l'éclairage et la suppression des ombres portées. Des hublots placés dans la coupole permettaient l'observation de l'opération depuis l'extérieur. Inscrit par arrêté du 23 avril 2012, ce bloc opératoire a été proposé au classement par la commission nationale des Monuments historiques, lors de sa séance du 16 mars 2015.

\section{Le cas particulier des lieux de mémoire}

Dans certains cas, minoritaires, le classement d'un établissement hospitalier a été justifié par l'importance de la mémoire qui s'y attache plutôt que par la qualité de son architecture.

Le poste de secours souterrain dit de Manonviller, en Meurthe-et-Moselle, en fait situé à Domjevin, a servi aux blessés jugés non transportables lors de la Première Guerre mondiale, et comprenait une salle d'opération. Il a été classé par arrêté du 10 février 1922.

38 L'ancienne maternité suisse d'Elne (Pyrénées-Orientales), installée dans un manoir préexistant, a été classée par arrêté du 4 mars 2013 essentiellement parce qu'elle a été le lieu de l'action humanitaire d'Élisabeth Eidenbenz, qui a permis de faire naître, entre 
décembre 1939 et avril 1944, dans des conditions satisfaisantes, des enfants dont les parents étaient pour la plupart des réfugiés espagnols, juifs et tsiganes. Depuis cette époque, le bâtiment où a été installée la maternité a été modifiée ${ }^{21}$.

\section{Les enjeux actuels de la protection du patrimoine hospitalier}

39 Le patrimoine hospitalier est sans doute l'une des catégories qui illustre le plus l'apparente contradiction entre les nécessités d'adaptation des immeubles à l'évolution de leur usage et les enjeux de conservation qui accompagnent la protection au titre des monuments historiques. Cette contradiction s'est exacerbée du fait de l'évolution accélérée des techniques et pratiques médicales tout au long $\mathrm{du} \mathrm{xx}^{\mathrm{e}}$ siècle, et particulièrement dans sa seconde moitié. Une personne, hospitalisée dans les années 1950 à l'hôpital parisien de Lariboisière - pourtant surnommé, à sa construction, "le Versailles de la misère" - nous décrivait encore un système de chambrées qui caractérisait la quasi-totalité des établissements hospitaliers jusqu'au début $\mathrm{du} \mathrm{xx}^{\mathrm{e}}$ siècle mais nous paraît aujourd'hui inenvisageable, car contraire aux plus élémentaires principes de confort, d'hygiène et d'intimité.

40 Le patrimoine hospitalier, pris au sens large, représente un corpus considérable en nombre, mais aussi, pour la plupart, en surface et en volume de bâtiments, situés au cœur d'une ville ancienne, ou rattrapés par l'urbanisation aux XIX et $\mathrm{XX}^{\mathrm{e}}$ siècles.

\section{Les modifications de l'organisation hospitalière française}

41 La conception et la localisation de nombre d'établissements hospitaliers anciens sont aujourd'hui obsolètes, après une stabilité souvent multiséculaire. En témoignent les projets de regroupement d'Orléans et de Dijon, qui verront la fermeture de grands établissements, ou ceux qui ont récemment affecté certains hôpitaux parisiens, ou encore l'hôtel-Dieu de Lyon. De même, de nombreux établissements de villes moyennes ou petites sont condamnés par les modifications de la «carte hospitalière ", comme le sont en même temps d'autres sièges d'institutions publiques, notamment des palais de justice ou des prisons.

42 Ces restructurations représentent une menace pour la conservation de ces ensembles, qui a conduit le ministère de la Culture, dans l'instruction du $1^{\mathrm{er}}$ décembre 2008 sur la protection des immeubles, à inscrire celle des établissements hospitaliers au nombre des priorités de la politique des monuments historiques.

Que protéger? Dès lors que l'ensemble immobilier présente un intérêt suffisant ou public, pour reprendre les termes du Code, il convient de le protéger le plus largement possible, en expliquant au propriétaire, personne publique exploitante ou vendeuse et/ ou personne privée acquéreuse, que la protection ne fera pas nécessairement obstacle à l'aménagement de certaines parties mais que cet aménagement s'effectuera sous la surveillance du service pour qu'il soit le moins destructif possible. Cette protection large facilitera, y compris pour le propriétaire, l'appréhension et la gestion globale des projets, et garantira la prise en compte de l'intérêt patrimonial de l'ensemble des éléments protégés par les maîtres d'œuvre. 

projets de réaménagement éventuels ne soient trop avancés, ou que leur adaptation n'entraîne des charges financières trop lourdes. Cela suppose un important travail de conviction, car il est généralement illusoire d'espérer protéger (même au niveau de l'inscription) un ensemble hospitalier contre l'avis de la collectivité et de l'établissement. Il s'agit donc d'évaluer avec eux les conséquences de la protection, en leur montrant ce qui constitue le "point dur ", les éléments à conserver dans leur intégrité, et ce qui pourra évoluer, ne serait-ce que par «habillage » intérieur des volumes.

\section{La nécessaire adaptation aux évolutions de la pratique hospitalière} (nôpital a été conçu par Tony Garnier. La première pierre fut posée en 1913 mais l'ensemble fut inauguré seulement en 1933. Nous sommes ici à la fin d'un processus qui, après le plan en peigne, visait à privilégier au niveau du sol un plan pavillonnaire sans galeries extérieures. Toutefois, les pavillons en béton armé, à étages couverts de terrasses, étaient reliés par un réseau de galeries souterraines ${ }^{22}$.

entendons bien qu'il s'agit d'un exemple concernant un ensemble immobilier inscrit, qui n'a pas été proposé au classement par la commission régionale. Il n'est toutefois pas déraisonnable de penser que cet ensemble mériterait une telle mesure de protection. Quoi qu'il en soit, cet exemple illustre bien les exigences contradictoires des préoccupations patrimoniales et de la santé publique, qui sont à la source de compromis. En effet, l'hôpital a été inscrit par arrêté du 20 novembre 2006, à l'exception de certains pavillons que l'administration hospitalière se réservait le droit de démolir dans le cadre de la modernisation de l'hôpital. Il va sans dire que c'est l'ensemble qui mérite une protection au titre des monuments historiques. Le projet, qui semblait avoir été abandonné, a resurgi pour les quatre pavillons non inscrits. Cette affaire est suivie de très près par le service des Monuments historiques. Les Hospices civils de Lyon, arguant de la nécessité de ne pas entraver le bon fonctionnement de l'hôpital, ont finalement obtenu la démolition de l'un des quatre pavillons. Il a cependant été demandé que soient préservés les trois autres pavillons non protégés, et restaurés les édifices protégés au titre des monuments historiques. La conservation régionale des Monuments historiques et le service territorial de l'Architecture et du Patrimoine du Rhône ont veillé à ce que la nouvelle construction soit en harmonie avec l'ensemble, par le biais de la législation sur les abords.

\section{La reconversion des établissements désaffectés}

En cas de désaffectation des établissements hospitaliers, une protection au titre des monuments historiques peut apparaitre comme un obstacle aux projets d'éventuels repreneurs visant une opération immobilière.

Le cas de l'hôpital Saint-Charles de Montpellier est particulièrement intéressant. Il comprend notamment l'ancien hôpital général des $\mathrm{XVII}^{\mathrm{e}}$ et $\mathrm{XVIII}^{\mathrm{e}}$ siècles et les cliniques 
Saint-Charles, construites dans la première moitié du $\mathrm{xx}^{\mathrm{e}}$ siècle. L'importance des cliniques Saint-Charles est réelle. Elles sont l'œuvre des architectes Paul Pelletier et Arthur Teisseire, et furent élevées entre 1932 et 1939. L'architecture est un exemple précoce de l'hôpital-bloc, issu des États-Unis, et qui se développe en France à partir environ de 1930, après l'abandon du système pavillonnaire. Les cliniques se rattachent à l'esthétique Art déco, avec deux bas-reliefs monumentaux de Joachim Costa et des vitraux d'Émile Brière. La chapelle de l'hôpital général est classée depuis le $1^{\mathrm{er}}$ avril 1947. Le 7 août 1963, l'escalier avec le vestibule adjacent sont inscrits. Dès 1993, le bâtiment d'ophtalmologie de 1892 est détruit, et les bâtiments sont progressivement désaffectés. L'ensemble devait être vendu pour financer le plan directeur du CHU. L'extension de protection qui est alors envisagée fut jugée susceptible de décourager d'éventuels repreneurs et de faire baisser le cours de la vente. Le 29 avril 1997, la COREPHAE se prononce pour l'inscription de la totalité des bâtiments anciens de l'hôpital général et des vestiges d'un asile d'aliénés construit entre 1821 et 1824 selon les prescriptions d'Esquirol. Elle se prononce également pour le classement de la totalité des cliniques Saint-Charles (bâtiment principal, jardins et pavillons d'entrée). Le 29 septembre 1997, la commission supérieure des Monuments historiques reprend à son compte, dans un premier temps, l'avis favorable à une inscription en totalité de l'ensemble hospitalier, non prise entre-temps par le préfet de région (hôpital général, vestiges de l'asile d'aliénés et cliniques Saint-Charles avec le jardin et les deux pavillons d'entrée). Dans un deuxième temps, la commission se prononce en faveur du classement des façades et toitures et des escaliers de l'hôpital général, des façades et toitures des bâtiments subsistant de l'asile, ainsi que des façades et toitures, des escaliers et du vestibule du bâtiment des cliniques, avec le jardin et les façades et toitures des deux pavillons d'entrée. La commission s'est en outre résolue à une procédure de classement d'office, si nécessaire.

50 En fait, seule fut prise l'inscription, par arrêté du 13 novembre 1997, des bâtiments de l'hôpital général, et du bâtiment des cliniques avec ses pavillons d'entrée et son jardin, à l'exclusion de certaines adjonctions. À partir d'un contexte un peu tendu, on a finalement abouti à une situation médiane. Des parties du $\mathrm{XIX}^{\mathrm{e}}$ siècle, dont les vestiges de l'asile d'aliénés, ont été démolies, et les cliniques Saint-Charles conservées pour être transformées en logements. Une voie nouvelle les sépare de l'ancien hôpital général, dont la partie du XvII ${ }^{e}$ siècle est occupée par un centre universitaire de recherches. En outre, la parcelle a été densifiée ${ }^{23}$.

51 La désaffectation d'un ensemble peut être au contraire l'occasion de clarifier et d'homogénéiser les protections existantes. Ce fut le cas récemment pour l'hôtel-Dieu de Lyon $^{24}$, classé en totalité par arrêté du 22 novembre 2011, mesure d'autant plus opportune qu'après l'avoir désaffecté, les Hospices civils de Lyon, propriétaires, ont passé un bail à construction pour la réalisation d'un hôtel, de commerces et de locaux pour des activités relevant du tertiaire. Trois phases de classement avaient depuis 1939 porté sur les éléments les plus remarquables, en délaissant la grande majorité des intérieurs et toute la partie du XIX ${ }^{e}$ siècle. Ce classement en totalité permet, d'un point de vue scientifique, de prendre en compte le développement progressif d'un ensemble hospitalier autour de quelques points forts, les différentes parties étant étroitement liées.

52 À l'occasion de la désaffectation de l'hospice de la Charité à Mâcon (Saône-et-Loire), celui-ci a été classé par arrêté du 28 juin 2013. Nous y retrouvons l'architecte Soufflot, 
qui fournit les plans pour sa construction, à l'emplacement d'une fondation de saint Vincent de Paul ${ }^{25}$.

La désaffectation de l'hôpital Laënnec à Paris n'a pas abouti à une telle homogénéisation des protections existantes par un classement global. La commission supérieure des Monuments historiques avait pourtant souhaité, entre autres, le classement de toutes les façades et toitures des bâtiments des XVII ${ }^{\mathrm{e}}$ et XVIII ${ }^{\mathrm{e}}$ siècles et des sols mais son avis n'a pas été suivi ${ }^{26}$.

\section{Conclusion}

Comme nous venons de le voir, le champ des établissements hospitaliers visés par les mesures de classement s'est diversifié, comme d'ailleurs pour tous les types de patrimoine, les constructions médiévales et les parties d'édifices les plus remarquables étant au départ privilégiées. $\mathrm{Si}$, au fil des protections, nous avons plusieurs exemples pour la période allant du Moyen Âge à la Révolution, les établissements des XIX et du $\mathrm{XX}^{\mathrm{e}}$ siècle sont sous-représentés.

Certaines catégories d'édifices sont absentes du corpus des édifices classés. Si nos renseignements sont exacts, aucun établissement voué à la lutte contre la tuberculose (hôpitaux marins et sanatoriums) n'est classé. Quelques-uns sont inscrits (l'ancien sanatorium d'Aincourt dans le Val-d'Oise ${ }^{27}$ en 1999, l'hôpital-sanatorium Sabourin à Clermont-Ferrand dans le Puy-de-Dôme ${ }^{28}$ et l'ancien aérium d'Arès en Gironde ${ }^{29}$ en 2000, le sanatorium Martel de Janville à Passy en Haute-Savoie ${ }^{30}$ en 2008). Comme le prouve l'état d'abandon de l'établissement d'Aincourt, il se pose pour ces ensembles des problèmes de réutilisation. L'hôpital-sanatorium Sabourin, à Clermont-Ferrand a cependant fait l'objet de travaux afin d'accueillir une école d'architecture, inaugurée le 16 octobre 2015. Dans ce cas, les dispositions d'origine ont été conservées, essentiellement en ce qui concerne les extérieurs ${ }^{31}$.

Par ailleurs, la politique patrimoniale s'avère complexe. Si les édifices concernés, appelés à s'adapter aux évolutions de leur mission primordiale, sont en activité, le dialogue est essentiel. Une bonne anticipation des événements est nécessaire en cas de désaffectation, qui peut être l'occasion d'une remise à plat des protections.

\section{Et le mobilier?}

Nous terminerons par une évocation de la question du décor et du mobilier, qui pose des problèmes particuliers. Le patrimoine mobilier hospitalier a été d'ailleurs le sujet du colloque organisé par l'association des conservateurs des antiquités et objets d'art de France en Bourgogne, en 2002.

Les chapelles d'hôpitaux sont au cœur de ces problématiques, comme l'a récemment démontré l'affaire de la chapelle de l'hôpital Laënnec à Paris. En effet, les repreneurs privés d'anciens établissements hospitaliers ne sont pas toujours désireux de conserver les chapelles (qui, rappelons-le, ne sont pas affectées au culte au sens des lois de séparation de 1905 et 1907) dans leur aménagement et leur décor historiques, et pour tout dire, se demandent parfois qu'en faire. D'autre part, le Code général de la propriété des personnes publiques assimile aujourd'hui les objets mobiliers classés et inscrits au titre des monuments historiques au domaine public des collectivités propriétaires, ce 
qui les rend inaliénables au profit d'une personne privée, à moins d'un déclassement ou d'une radiation de l'inscription préalable. Les objets mobiliers protégés d'une chapelle hospitalière ne peuvent ainsi être cédés au repreneur privé de l'ensemble immobilier, et ne peuvent y demeurer que moyennant un dépôt de la personne publique qui le lui a vendu.

La question du maintien dans les lieux se pose d'ailleurs, car si le nouveau propriétaire n'envisage pas d'ouverture au public, il peut sembler préférable à la personne publique vendeuse de déménager les œuvres et de les présenter en un autre lieu. Dans le cas d'une apothicairerie, faut-il « sortir » les objets de pharmacie de leur cadre menuisé, ou bien démonter celui-ci et transférer le tout en un autre lieu? Cette solution, sans doute satisfaisante du point de vue du maintien de la cohérence de l'ensemble et de sa présentation au public, n'est-elle pas en contradiction avec notre sacro-saint principe de conservation in situ? C'est la question qui s'est récemment posée pour l'apothicairerie de l'hôpital général de Dijon.

60 Par ailleurs, la politique patrimoniale s'avère complexe. Si les édifices concernés, appelés à s'adapter aux évolutions de leur mission primordiale, sont en activité, le dialogue est essentiel. Une bonne anticipation des événements est nécessaire en cas de désaffectation, qui peut être l'occasion d'une remise à plat des protections. Enfin, l'article L. 622-1-2. de la loi du 7 juillet 2016, relative à la liberté de la création, à l'architecture et au patrimoine, prévoit la possibilité de créer une servitude de maintien dans les lieux pour les objets mobiliers classés ou les ensembles historiques mobiliers classés, lorsqu'ils sont attachés par des liens historiques ou artistiques remarquables, à un immeuble classé. Ces dispositions ont naturellement vocation à s'appliquer également au patrimoine hospitalier.

61 Comme celle des palais de justice ou des prisons, la réorganisation de la carte hospitalière amène beaucoup de questions, qui interrogent nos pratiques de protection. Il ne semble pas exister de réponse générale à ces questions.

\section{NOTES}

1. - Voir dans la base Mérimée : notice PA00104846.

2. - Voir dans la base Mérimée : notice PA00097358.

3. - Voir dans la base Mérimée : notice PA00078266.

4. - Voir dans la base Mérimée : notice PA00082006.

5. - Voir dans la base Mérimée : notice PA00082577.

6. - Voir dans la base Mérimée : notice PA00096684.

7. - Voir dans la base Mérimée : notice PA00112275.

8. - Voir dans la base Mérimée : notice PA00113229.

9. - Voir dans la base Mérimée : notice PA00094529.

10. - Voir dans la base Mérimée : notice PA00114515.

11. - Voir dans la base Mérimée : notice PA00086494.

12. - Voir dans la base Mérimée : notice PA72000015. 
13. - L'hôpital Lariboisière à Paris, élevé par Martin-Pierre Gauthier d'après un plan approuvé en 1845, sur un schéma en peigne, avec des galeries couvertes seulement au rez-de-chaussée, fut le premier de ce type à acquérir une grande notoriété dépassant les frontières. Actuellement, il est inscrit par arrêté du 15 janvier 1975 pour ses façades et toitures, ses galeries intérieures et sa chapelle.

14. - Voir dans la base Mérimée : notice PA00079904.

15. - Voir dans la base Mérimée : notice PA29000020.

16. - Voir dans la base Mérimée : notice PA00135687.

17. - Voir dans la base Mérimée : notice PA00105912.

18. - Voir dans la base Mérimée : notice PA00105930.

19. - Voir dans la base Mérimée : notice PA50000056.

20. - Voir DREYER, Francis. " Histoire de l'hôpital. L'éclairage des salles d'opération aux XIX et $X^{\mathrm{e}}$ siècles : l'apparition du scialytique ", In Situ [En ligne], 10 | 2009, mis en ligne le 19 mai 2009, consulté le 03 janvier 2017. URL : http://insitu.revues.org/3997 ; DOI : 10.4000/insitu.3997.

21. - Voir dans la base Mérimée : notice PA66000037. Voir dans ce numéro : Michèle François. « La maternité suisse d'Elne (Pyrénées-Orientales) 1939-1944 », In Situ [En ligne], 31|2017, mis en ligne le 23 février 2017, consulté le 28 février 2017. URL : http://insitu.revues.org/14158.

22. - Voir le très complet dossier électronique d'inventaire : http://patrimoine.rhonealpes.fr/ dossier/hopital-edouard-herriot/fe9e1af3-a15b-41a4-b009-70dfc4a85c32.

23. - Voir dans la base Mérimée : notice PA00103544. Voir dans ce numéro: Nicolas Morestin. "L'îlot Saint-Charles à Montpellier: un triple programme de réhabilitation du patrimoine hospitalier ", In Situ [En ligne], 31 |2017, mis en ligne le 23 février 2017, consulté le 28 février 2017. URL : http://insitu.revues.org/14179.

24. - Voir dans la base Mérimée : notice PA00117821. Voir dans ce numéro : Véronique Belle. « La notion de patrimoine à travers trois liasses du fonds des Hospices civils de Lyon : la bataille de l'hôtel-Dieu ", In Situ [En ligne], 31 | 2017, mis en ligne le 22 février 2017, consulté le 28 février 2017. URL : http://insitu.revues.org/14119.

25. - Voir dans la base Mérimée : notice PA00113322.

26. - Voir dans la base Mérimée : notice PA00088690.

27. - Voir dans la base Mérimée : notice PA95000005.

28. - Voir dans la base Mérimée : notice PA63000030.

29. - Voir dans la base Mérimée : notice PA33000023.

30. - Voir dans la base Mérimée : notice PA74000012.

31. - Voir dans ce numéro: Philippe Grandvoinnet. "Valoriser le patrimoine climatique : la reconversion des sanatoriums de cure antituberculeuse », In Situ [En ligne], 31|2017, mis en ligne le 23 février 2017, consulté le 28 février 2017. URL : http://insitu.revues.org/14173.

\section{RÉSUMÉS}

Comme cela a été le cas pour les autres types de patrimoine, la protection au titre des monuments historiques du patrimoine immobilier hospitalier s'est élargie au fil du temps, depuis la mention de "l'hôtel-Dieu » d'Angers dans la première liste des monuments historiques de 1840. Au moment de la publication de la loi du 31 décembre 1913 sur les monuments historiques, ce sont surtout les constructions médiévales qui avaient été prises en compte, en distinguant les 
éléments les plus remarquables aux dépens de l'organisation d'ensemble. L'évolution est allée dans le sens de protections plus globales et d'un élargissement typologique et chronologique. Aujourd'hui encore, si l'on ne considère que le corpus des immeubles classés, le patrimoine hospitalier du XIXe siècle, et a fortiori celui du $\mathrm{XX}^{\mathrm{e}}$ siècle, est peu représenté. Les protections futures devront tenir compte de la réorganisation sans précédent de la carte hospitalière, et pour les établissements protégés toujours en activité, de l'évolution des pratiques hospitalières. Le dialogue est une des conditions essentielles d'une protection satisfaisante du patrimoine hospitalier: l'administration hospitalière craint, dans certains cas, que la protection patrimoniale ne soit en contradiction avec l'évolution des équipements de soins ou qu'elle constitue, en cas de désaffectation des établissements hospitaliers, un obstacle aux projets d'éventuels repreneurs.

As for other kinds of built heritage, the historic monuments protection of hospitals has evolved considerably since the earliest protection, that of the Angers Hôtel-Dieu, mentioned on the first list of historic monuments in France, in 1840. When the new historic monuments law of 31 December 1913 was passed, it was largely medieval hospital establishments that had been given statutory protection, often distinguishing spectacular individual constructions at the expense of the hospital organisation as a whole. The trend however has been towards more coherent global protection measures, and also to the inclusion of a broader typological and chronological range of hospital buildings. Today, however, if we limit ourselves to the monuments which are 'classés', nineteenth and, even more, twentieth-century hospitals are still poorly protected. Hospital protections in the future will have to take into account the radical reorganisation of the country's hospital infrastructure, and, if the protection is given to functioning hospitals, it will have to take into account the evolution of hospital practices. For a satisfactory protection of the hospital heritage, dialogue is a key consideration. The hospital administration is often wary of historic monument protection, fearing that it will be in contradiction with the way equipment must evolve, or, if the hospital is closed down, that it will discourage potential buyers.

\section{INDEX}

Keywords : apothecary store, asylum, Esquirol (Jean-Étienne), Garnier (Tony), hospital, hospice, Hôtel-Dieu, historic monument, Nelson (Paul), sick wards, operating theatre, sanatorium, Soufflot (Jacques-Germain), Tollet (Casimir)

Mots-clés : apothicairerie, asile, Esquirol (Jean-Étienne), Garnier (Tony), hôpital, hospice, hôtelDieu, monument historique, Nelson (Paul), salle des malades, salle d'opération, sanatorium, Soufflot (Jacques-Germain), Tollet (Casimir)

\section{AUTEURS}

\section{DOMINIQUE PERRIN}

Adjoint au chef du bureau de la protection des monuments historiques, Sous-direction des monuments historiques et des espaces protégés, direction générale des Patrimoines, ministère de la Culture et de la Communication dominique.perrin@culture.gouv.fr

\section{FRANTZ SCHCENSTEIN}

Chef du bureau de la conservation du patrimoine immobilier, Sous-direction des monuments historiques et des espaces protégés, direction générale des Patrimoines, ministère de la Culture et de la Communication frantz.schoenstein@culture.gouv.fr 